\title{
Editorial
}

\section{Predicting ischaemic events in the periopera- tive period: in search of the perfect tool}

Jean-Claude Tardif MD FRCPC, Martin Juneau MD FRCPC

siderable interobserver variability in the interpretation of coronary angiograms represented another limitation. ${ }^{10}$ Although the advent of quantitative coronary angiography has reduced the variability of the technique, it has not improved the ability to evaluate the physiological significance of intermediate lesions.

Diagnostic methods to assess the physiological relevance of coronary narrowing were developed. Myocardial lactate production was measured during periods of increased oxygen demand, ${ }^{1}$ but this technique requiring coronary sinus catheterization has fallen out of interest. Determination of coronary flow reserve, defined as the ratio between hyperaemic and baseline flow, then became possible. Early assessment of the absolute coronary flow reserve was performed after transient arterial occlusion and measurement of hyperemic flow with an electromagnetic flowmeter. ${ }^{12}$ It was observed that normal coronary arteries could increase their flow fourfold during hyperaemia, whereas stenosed vessels lost that ability. However, this specific method obviously could not be used clinically.

Positive-emission tomography (PET) and nuclear scanning were developed and allow assessment of absolute and relative coronary flow reserve, respectively. Both methods, however, also have limitations for the prediction of perioperative coronary events. Although PET scanning is a very powerful research tool, it suffers from its lack of availability. Furthermore, absolute flow reserve can be altered by factors other than arterial narrowings, such as haemodynamic changes or ventricular hypertrophy. ${ }^{13}$ Although the diagnostic value of nuclear scanning has been validated, controversy exists as to its ability to predict cardiac events in patients undergoing major noncoronary surgical revascularization procedures. $^{14}$

The observation that regional wall motion abnormalities appear very early during myocardial ischaemia was the basis for the development of exercise echocardiography. ${ }^{15}$ The diagnostic value of stress transthoracic 
echocardiography for coronary artery disease has been shown to be similar to that of nuclear scanning, ${ }^{16}$ and its ability to identify patients at risk for perioperative coronary events has been demonstrated. ${ }^{17}$ The positive predictive value, however, is rather low and comparable to dipyridamole thallium scintigraphy so that most patients with an abnormal echocardiographic or scintigraphic result can undergo surgery without experiencing hard coronary events. The important question of what constitutes the optimal strategy for patients with abnormal test results has not been decisively answered. Additional limitations of stress echocardiography are the occasional sub-optimal image quality, its operator-dependence, and its nonquantitative analysis.

\section{Newer diagnostic methods}

The advent of intravascular ultrasound (IVUS), which uses one or more miniaturized transducers at the tip of catheters to provide cross-sectional images of coronary arteries, has dramatically changed our understanding of the limitations of angiography as a gold standard. ${ }^{18}$ Indeed angiography, which displays a two-dimensional sithouette of the complex tridimensional arterial lumen, is sub-optimal compared with the tomographic views provided by IVUS.

Intravascular ultrasound was developed to supply detailed information not only on arterial stenosis, but also on atherosclerotic lesions and on the vessel wall. Depiction of the coronary lumen, and not the wall, is an important limitation of angiography, atherosclerosis being primarily a disease of the arterial wall. The extent and severity of coronary artery disease is considereably underestimated by angiography. Half of patients with angiographically mildly diseased coronary arteries have narrowings of $50 \%$ or more with IVUS. ${ }^{19}$ Moreover, the majority of angiographically normal reference segments are involved in the diffuse atherosclerotic process on IVUS imaging, which contributes to the angiographic underestimation of stenoses. Arterial remodeling, which refers to compensatory enlargement of the external elastic membrane that develops to limit lumen obtruction despite increasing plaque burden, represents another factor responsible for the angiographic underestimation. ${ }^{20}$ Finally, the composition and morphology of atherosclerotic lesions, which can be assessed with IVUS but not with angiography, are major determinants of their clinical risk. ${ }^{21}$ Although IVUS clearly augments angiography, it nevertheless shares the limitation of assessing anatomy and not physiology.

Intracoronary Doppler, which consists in measuring baseline and hyperaemic velocities with a flowire in the coronary artery, was developed to allow determination of flow reserve in the catheterization laboratory, thus permitting assessment of anatomy and physiology in a single procedure. ${ }^{22}$ Intracoronary Doppler and dipyridamole scintigraphy have recently been shown to correlate better with each other than with angiography in patients with lesions of intermediate severity. ${ }^{23}$ Nevertheless, Doppler results may vary with changes in arterial pressure, and this limitation has been the impetus for the recent work on fractional flow reserve, which is based on measurement of the hyperaemic translesional pressure gradient. ${ }^{24}$ Determination of fractional flow reserve also requires placement of a guidewire in the coronary artery, and it is not yet a routine clinical tool as it has been used only by a small number of investigators.

Thus, all of our diagnostic tools to assess coronary artery disease have limitations. The problem of predicting perioperative ischaemic complications is compounded by the fact that the majority of myocardial infarctions are caused by stenoses less than $50 \%$ on a previous angiogram. ${ }^{25}$ Furthermore, the risk of plaque rupture and of an acute coronary event may be affected by the severity of endothelial dysfunction, which is not specifically assessed by any of the previously discussed methods. Finally, the presence of haemodynamic instability that occasionally may trigger myocardial ischaemia in the operating room depends on factors other than the severity of coronary artery disease.

\section{Detection of perioperative myocardial ischaemia}

Detecting myocardial ischaemia in the operating room appears at first an easier task than predicting its occurrence. However, the important limitations of electrocardiographic monitoring for the detection of myocardial ischaemia are discussed in Dr. Yang's elegant article, ${ }^{7}$ which focuses on ST segment analysis. Ischaemic ECG changes can also be manifested by $\mathrm{T}$ wave abnormalities, but the interpretation of any ECG changes in the perioperative period remains difficult, especially after cardiac surgery as was noted by Dr. Ansley et al. in their work also published in this issue of the Journal. ${ }^{26}$ Concordance between the presence of perioperative ST segment abnormalities and regional wall motion dysfunction on transoesophageal echocardiography (TEE) is far from being perfect. ${ }^{27,28}$ Continuous monitoring cannot be considered a gold standard for identifying myocardial ischaemia in this setting and the article by Dr. Ansley et al. therefore represents a comparison of systems for the detection of ECG changes and not of myocardial ischaemia per se. Nevertheless, their findings will be useful for clinicians and possibly for the development of improved monitors for the operating room.

Transoesophageal echocardiography presently is the 
most reliable and practical method to detect myocardial ischaemia in the operating room. Prolonged postoperative TEE monitoring is more difficult in the intensive care unit. Importantly, appropriate training in echocardiography is necessary as experience and skill are required for the performance of a reliable TEE examination. Asynchrony of contraction of the interventricular septum that commonly occurs after cardiac surgery represents a minor limitation to the assessment of segmental function with TEE, and should not be confused with ongoing myocardial ischaemia. Also, distinction between stunned and ischaemic myocardium may be impossible with transoesophageal examination.

The positive predictive value of TEE for a perioperative cardiac event, is low (33\% or less). ${ }^{27,29} \mathrm{~A}$ similar situation exists with ECG monitoring, with only $7 \%$ of patients with postoperative ST segment abnormalities experiencing ischamic coronary events. ${ }^{30}$ The question of whether such monitoring is justified will be easier to answer when trials have determined whether prevention or early treatment of perioperative ischaemia can reduce the incidence of acute coronary events.

\section{Prédiction des événe- ments ischémiques périopératoires: à la recherche de l'instrument parfait}

Pendant des années, l'évaluation des patients souffrant d'insuffisance coronaire a été relativement simple. L'angine était identifiée lorsque la douleur thoracique se manifestait à l'effort et signifiait une diminution importante du calibre des artères coronaires épicardiques. ${ }^{1} \mathrm{La}$ standardisation de l'épreuvre d'effort avec électrocardiogramme a favorisé son utilisation généralisée autant pour le diagnostic que le pronostic. ${ }^{1}$ Malheureusement, le syndrome angineux et son évaluation se sont avérés beaucoup plus compliqués par la suite. On a réalisé que des épisodes d'ischémie myocardique pouvaient survenir sans douleur thoracique et que l'ischémie silencieuse influençait le pronostic. ${ }^{3}$ On distinguait en outre une variante de l'angine survenant surtout au repos et causée par un vasospasme. ${ }^{4}$ Il devenait également évi- dent que la physiopathologie de l'angine instable et de l'infarctus du myocarde comportait non seulement un vasospasme mais aussi une rupture de plaque ainsi que la formation d'un thrombus. Il devenait aussi évident que l'insuffisance coronaire ne résultait pas simplement de l'accumulation progressive de matériel athérosclérotique. ${ }^{5} \mathrm{~L}$ importance d'un désordre endothélial coronarien favorisant la rupture de la plaque et le rôle de l'adhésion plaquettaire et de la formation de thrombus étaient aussi reconnus. ${ }^{6}$ Comme le décrit l'article du Dr Yang dans ce numéro du Journal, on réalisait enfin que l'épreuvre d'effort était limitée. ${ }^{7}$

\section{Évaluation anatomique et physiologique de l'insuffisant coronarien}

C'est grâce à plusieurs outils diagnostiques récents que nos connaissances cliniques et physiopathologiques ont pu évoluer. L'angiographie coronarienne sélective permettait aux cliniciens de diagnostiquer la présence et d'évaluer l'importance d'une sténose. ${ }^{8}$ Aux extrêmes de l'échelle clinique, on pouvait établir la gravité d'une sténose coronaire mais on connaissait peu l'importance des lésions de gravité intermédiaire sur l'hémodynamique. ${ }^{9}$ L'interprétation de la coronarographie de façon subjective telle que pratiquée en clinique montre une importante variabilité interobservateur, ce qui limite son utilité en recherche. ${ }^{10}$ Bien que l'introduction de l'angiographie coronaire quantitative ait réduit son inconstance, cette technique n'a pas amélioré notre capacité d'évaluer l'importance sur le plan physiologique des lésions intermédiaires.

Des épreuves diagnostiques ont été élaborées pour évaluer la gravité de la sténose coronaire. On a mesuré la production de lactate myocardique pendant des périodes au cours desquelles la demande en oxygène augmentait, " mais comme cette technique requiert la canulation du sinus coronaire, elle n'a pas connu de succès. Déterminer le débit coronaire de réserve, défini comme la relation entre le débit hyperhémique et le débit de base, était réalisable. On a d'abord mesuré le débit coronaire de réserve absolu après une occlusion artérielle transitoire et ensuite le débit en hyperhémie à l'aide d'un débitmètre électromagnétique. ${ }^{12}$ On savait que les artères coronaires normales pouvaient quadrupler leur débit pendant l'hyperhémie, alors que les vaisseaux sténosés perdaient cette habileté. Il était toutefois évident que cette technique ne pouvait être utilisée en clinique.

La tomographie par émission de positrons (PET) et la scintigraphie nucléaire permettent l'évaluation respective du débit coronaire de réserve absolu et relatif. Cependant, lorsqu'il s'agit de prédire les incidents coronaires périopératoires, ces deux méthodes sont insuffi- 
santes. Bien que PET représente un outil de recherche puissant, sa disponibilité est limitée. En outre, le débit de réserve absolu peut être modifié par d'autres facteurs que le rétrécissement de la lumière artérielle, comme les dérangements hémodynamiques et l'hypertrophie ventriculaire. ${ }^{13}$ Bien que la valeur diagnostique de la scintigraphie nucléaire ait été validée, on n'est pas d'accord sur sa capacité de prévoir les incidents cardiaques chez des patients soumis à des interventions de revascularisation non coronariennes. ${ }^{14}$

L'observation de l'apparition précoce des anomalies de la motilité de la paroi pendant l'ischémie myocardique a été à la base du développement de l'échocardiographie d'effort. ${ }^{15}$ On a démontré que la valeur diagnostique de l'échocardiographie transthoracique d'effort était identique à celle de la scintigraphie nucléaire ${ }^{16}$ et qu'elle pouvait identifier les patient à risque de complications coronaires. ${ }^{17} \mathrm{La}$ valeur prédictive positive est cependant plutôt faible et comparable à celle de la scintigraphie au dipyridamole thallium de sorte que la plupart des patients dont l'échocardiographie ou la scintigraphie est anormale subissent généralement une intervention sans complications. La stratégie optimale à adopter en face d'un patient dont les épreuves sont anormales est une question à laquelle on n'a pas encore trouvé de réponse. Les autres réserves propres à l'échocardiographie d'effort sont constituées par la pauvreté occasionnelle de l'image, sa dépendance de l'échocardiographiste et son analyse non quantitative.

\section{Les nouvelles méthodes diagnostiques}

L'avènement de l'ultrasonographie intravasculaire (USIV) qui utilise un ou plusieurs transducteurs miniaturisés à l'extrémité d'un cathéter pour fournir des images transversales des artères coronaires a dramatiquement modifié notre perception des faiblesses de la coronarographie considérée alors comme la norme. ${ }^{18} \mathrm{La}$ coronarographie qui ne procure de la lumière artérielle, qu'une silhouette en deux dimensions d'un complexe tridimensionnel est donc inférieure à la représentation tomographique fournie par l'USIV.

L'ultrasonographie intravasculaire n'a pas été développée pour donner uniquement des renseignements précis sur la sténose artérielle mais aussi sur les lésions d'athérosclérose et sur la paroi du vaisseau. La description de la lumière artérielle sans un aperçu de la paroi limite donc considérablement la coronarographie, car l'athérosclérose est d'abord une affection de la paroi. La coronarographie sous-estime fortement l'étendue et la gravité de l'insuffisance coronaire. La moitié des sténoses jugées légères à la coronarographie sont des rétrécissements de $50 \%$ ou plus à l'USIV. ${ }^{19}$ En outre, la majorité des segments-repères trouvés normaux à la coronarographie paraissent atteints par un processus athérosclérotique diffus à l'imagerie USIV, ce qui démontre la sous-évaluation angiographique des sténoses. Le remodelage artériel est constitué par la dilatation compensatoire qui évolue pour restreindre l'obstruction de la lumière artérielle malgré l'augmentation de la densité des plaques; le remodelage représente un autre facteur responsable de la sous-évaluation angiographique. ${ }^{20}$ Finalement, la composition et la morphologie des plaques athérosclérotiques qui peut être déterminée par I'USIV mais non par la coronarographie, sont des déterminants majeurs du risque clinique. ${ }^{21}$ Bien que l'USIV soit de toute évidence supérieure à la coronarographie, elle partage les mêmes limitations en ce qu'elle évalue l'anatomie et non la physiologie.

Le Doppler intracoronarien qui consiste à mesurer avec un détecteur de débit la vélocité initiale et en hyperthémie, a été développé pour permettre de déterminer le débit de réserve au laboratoire d'hémodynamique; une seule épreuve permet d'évaluer l'anatomie et la physiologie. ${ }^{22}$ On a montré récemment que le Doppler intracoronarien et la scintigraphie au dipyridamole avaient une meilleure corrélation entre eux que l'angiographie chez les patients souffrants de lésion de gravité intermédiaire. ${ }^{23}$ Néanmoins, les résultats obtenus avec le Doppler intracoronarien peuvent varier avec les changements de pression et, d'après des travaux récents, cet inconvénient a un impact majeur sur le débit de réserve fractionné, qui est basé sur la mesure du gradient de pression translésionnel en hyperhémie. ${ }^{24}$ La mesure du débit de réserve fractionné nécessite l'introduction d'un mandrin filiforme dans l'artère coronaire et n'est pas encore un outil utilisé couramment, n'ayant été utilisé jusqu'à maintenant que par un petit nombre de chercheurs.

Tous nos instruments de diagnostic servant à l'évaluation de l'insuffisance coronaire ont leurs limitations. La difficulté de prédire l'ischémie périopératoire vient de ce que la majorité des infarctus est causée par une sténose inférieure à $50 \%$ constatée sur une coronarographie antérieure. ${ }^{25}$ D'ailleurs, au cours d'une attaque coronlearienne aiguë, le risque de rupture de plaque peut être affecté par la lésion endothéliale, laquelle ne peut être spécifiquement évaluée par aucune des méthodes mentionnées. Finalement, l'apparition d'une instabilité hémodynamique qui, à l'occasion, peut déclencher l'ischémie myocardique en salle d'opération, peut dépendre de facteurs indépendants de la gravité de l'insuffisance coronaire.

\section{La détection de l'ischémie myocardique périopératoire}

En salle d'opération, la détection de l'ischémie 
myocardique paraît au premier abord plus facile que la prédiction de son apparition. Cependant, en ce qui concerne la détection de l'ischémie myocardique, les limitations importantes inhérentes au monitorage électrocardiographique sont discutées dans l'article élégant du Dr Yang ${ }^{7}$ qui porte son attention sur l'analyse du segment ST. Les altérations ECG ischémiques peuvent aussi se manifester par des anomalies de l'onde $\mathrm{T}$, mais l'interprétation de tout changement ECG demeure difficile à la période périopératoire, surtout après une chirurgie cardiaque, comme le font remarquer de Dr Ansley et al. dans un article publié lui aussi dans ce numéro du Journal. ${ }^{25}$ La corrélation existant entre la présence d'anomalies du segment ST et le dysfonctionnement de la motilité de la paroi régionale sur l'échographie transoesophagienne (ETO) est loin d'être parfaite; ${ }^{27,28}$ l'article de Ainsley et al. établit plutôt une comparaison entre des systèmes appliqués pour détecter des changements ECG et non l'ischémie myocardique per se. Néanmoins, leurs constatations seront utiles aux cliniciens et serviront à l'amélioration éventuelle des moniteurs utilisés en salle d'opération.

L'échographie transoesophagienne est présentement la méthode la plus fiable et la plus pratique pour détecter l'ischémie en salle d'opération. Un monitorage par ETO prolongé est plus difficile à l'unité des soins intensifs. Essentiellement, une formation appropriée en échocardiographie est nécessaire et l'expérience et l'habileté sont requises pour réaliser un examen valable par ETO. L'asynchronisme de la contractilité septale interventriculaire qui survient fréquemment après une chirurgie cardiaque limite quelque peu l'évaluation de la fonction segmentaire à l'ETO et ne doit pas être confondue avec une ischémie myocardique active. Il faut savoir distinguer entre un myocarde «stunned » et un myocarde ischémique, ce qui peut être cependant impossible avec l'ETO.

La valeur prédictive positive de l'ETO au cours d'un incident cardiaque périopératoire est faible $(33 \%$ ou moins). ${ }^{27,29}$ Une situation identique existe avec le monitorage ECG: seulement $7 \%$ des patients qui présentent une anomalie du segment ST évoluent vers l'ischémie myocardique. ${ }^{30}$ On pourra décider plus facilement si un tel monitorage est justifié quand expérimentalement on aura déterminé si la prévention et le traitement précoce de l'ischémie périopératoire peuvent réduire l'incidence des crises ischémiques aiguës.

\section{References}

1 Campeau $L$. Grading of angina pectoris (Letter). Circulation 1976 ; 54: 522-3.

2 Theroux $P$, Waters DD, Halphen C, Debaisieux JC, Mizgala $H F$. Prognostic value of exercise testing soon after myocardial infarction. N Engl J Med 1979; 301:

341-5.

3 Gottlieb SO, Gottlieb SH, Achuff SC, et al. Silent ischemia on Holter monitoring predicts mortality in high-risk postinfarction patients. JAMA 1988; 259: 1030-5.

4 Miller D, Waters DD, Warnica W, Szlachcic J, Kreeft J, Theroux $P$. Is variant angina the coronary manifestation of a generalized vasospastic disorder. N Engl J Med 1981 304: 763-6.

5 Fuster V, Badimon L, Badimon JJ, Chesebro JH. The pathogenesis of coronary artery disease and the acute coronary syndromes. N Engl J Med 1992; 326: 242-50.

6 Meredith IT, Anderson TJ, Uehata A, Yeung AC, Selwyn $A P$, Ganz $P$. Role of endothelium in ischemic coronary syndromes. Am J Cardiol 1993; 72: 27C-32C.

7 Yang $H$. Intraoperative automated ST segment analysis. Can J Anaesth 1996; 43: 1041-51.

8 Bourassa MG, Lesperance J, Campeau L, Bois MA, Saltiel $J$. Selective coronary angiography using a percutaneous femoral technique. Can Med Assoc J 1970; 102: 170-7.

9 White $C W$, Wright $C B$, Doty $D B$, et al. Does visual interpretation of the coronary arteriogram predict the physiologic importance of a coronary stenosis? N Engl J Med 1984; 310: 819-24.

10 Marcus ML, Skorton DJ, Johnson MR, Collins SM, Harrison DG, Kerber RE. Visual estimates of percent diameter coronary stenosis: "a battered gold standard". J Am Coll Cardiol 1988; 11: 882-5.

11 Arbogast $R$, Bourassa $M G$. Myocardial function during atrial pacing in patients with angina pectoris and normal coronary arteriograms. Comparison with patients having significant coronary artery disease. Am J Cardiol 1973; 32: 257-63.

12 Gould KL, Lipscomb K, Hamilton GW. Physiologic basis for assessing critical coronary stenosis. Instantaneous flow response and regional distribution during coronary hyperemia as measures of coronary flow reserve. Am J Cardiol 1974; 33: 87-94.

13 Gould KL, Kirkeeide RL, Buchi M. Coronary flow reserve as a physiologic measure of stenosis severity. J Am Coll Cardiol 1990; 15: 459-74.

14 Baron JF, Mundler $O$, Bertrand $M$, et al. Dipyridamolethallium scintigraphy and gated radionuclide angiography to assess cardiac risk before abdominal aortic surgery. $\mathrm{N}$ Engl J Med 1994; 330: 663-9.

15 Ryan T, Vasey CG, Presti CF, O'Donnell JA, Feigenbaum $H$, Armstrong $W F$. Exercise echocardiography: detection of coronary artery disease in patients with normal left ventricular wall motion at rest. J Am Coll Cardiol 1988; 11 : 993-9.

16 Quinones MA, Verani MS, Haichin RM, Mahmarian JJ, Suarez J, Zoghbi WA. Exercise echocardiography versus 201TI single-photon emission computed tomography in 
evaluation of coronary disease. Analysis of 292 patients. Circulation 1992; 85: 1026-31.

17 Poldermans D, Fioretti PM, Forster T, et al. Dobutamine stress echocardiography for assessment of perioperative cardiac risk in patients undergoing major vascular surgery. Circulation 1993; 87: 1506-12.

18 Tardif JC, Pandian NG. Intravascular ultrasound imaging in peripheral arterial and coronary artery disease. Current Opinion in Cardiology 1994; 9: 627-33.

19 Porter TR, Sears $T$, Xie F, et al. Intravascular ultrasound study of angiographically mildly diseased coronary arteries. J Am Coll Cardiol 1993; 22: 1858-65.

20 Glagov S, Weisenberg E, Zarins CK, Stankunavicius $R$, Kolettis GJ. Compensatory enlargement of human atherosclerotic coronary arteries. N Engl J Med 1987; 316: 1371-5.

21 Hodgson JM, Reddy KG, Suneja R, Nair RN, Lesnefsky EJ, Sheehan $H M$. Intracoronary ultrasound imaging: correlation of plaque morphology with angiography, clinical syndrome and procedural results in patients undergoing coronary angioplasty. J Am Coll Cardiol 1993; 21: 35-44.

22 Doucette JW, Corl PD, Payne HM, et al. Validation of a Doppler guide wire for intravascular measurement of coronary artery flow velocity. Circulation 1992; 85: 1899-911.

23 Miller DD, Donohue TJ, Younis LT, et al. Correlation of pharmalogical $99 \mathrm{mTc}$-sestamibi myocardial perfusion imaging with poststenotic coronary flow reserve in patients with angiographically intermediate coronary artery stenoses. Circulation 1994; 89: 2150-60.

24 De Bruyne B, Bartunek J, Sys SU, Heyndrickx GR. Relation between myocardial fractional flow reserve calculated from coronary pressure measurements and exercise-induced myocardial ischemia. Circulation 1995; 92: 39-46.

25 Little WC, Constantinescu M, Applegate RJ, et al. Can coronary angiography predict the site of a subsequent myocardial infarction in patients with mild-tomoderate coronary artery disease? Circulation 1988; 78: 1157-66.

26 Ansley DM, O'Connor JP, Merrick PM, et al. On line STsegment analysis for detection of myocardial ischaemia during and after coronary revascularization. Can J Anaesth 1996; 43: 995-1000.

27 Ellis JE, Shah MN, Briller JE, Roizen MF, Aronson S, Feinstein $S B$. A comparison of methods for the detection of myocardial ischemia during noncardiac surgery: automated STsegment analysis sytems, electrocardiography, and transesophageal echocardiography. Anesth Analg 1992; 75: 764-72.

28 Eisenberg $M J$, London $M J$, Leung $J M$, et al. Monitoring for myocardial ischemia during noncardiac surgery. A technology assessment of transesophageal echocardiography and 12-lead electrocardiography. JAMA 1992; 268: $210-6$.
29 Leung JM, O'Kelly B, Browner WS, Tubau J, Hollenberg $M$, Mangano DT. Prognostic importance of postbypass regional wall-motion abnormalities in patients undergoing coronary artery bypass graft surgery. Anesthesiology 1989; 71: 16-25.

30 Mangano DT, Browner WS, Hollenberg $M$, London $M J$, Tubau JF, Tateo IM. Association of perioperative myocardial ischemia with cardiac morbidity and mortality in men undergoing noncardiac surgery. N Engl J Med 1990; 323: $1781-8$. 